\title{
Diversity, Distribution and Relative Abundance of Avian Fauna of Nansebo Forest, Southern Oromia, Ethiopia
}

\author{
Ziyad Jemal Husein ${ }^{1} \quad$ Mustefa Sultan Dalu ${ }^{2}$ \\ 1.Arsi Mountains National Park, Oromia Forest and Wildlife Enterprise, Asella, Ethiopia. \\ 2.Department of Forestry, College of Agriculture and Environmental science, Arsi University, Asella, Ethiopia
}

\begin{abstract}
Birds are one of the components of biodiversity. Avian community studies are effective tools for monitoring a forest ecosystem. The status of birds in the present study areas is very little known. Therefore, the objective of the study was to assess species composition and relative abundance of birds at Nansebo forest in southern Ethiopia. To this end, a stratified random sampling technique was employed. Accordingly, to collect data for the study, the whole habitat of the study area was divided into dominant vegetation types. Accordingly, Moist Afromontane forest and modified habitat were identified in Nansebo forest. A line transects count aided by binocular was employed to investigate avian species diversity and relative abundance. Accordingly, 20 transect lines of $1 \mathrm{~km}$ with a width of $0.25 \mathrm{~km}$ or less was used to cover $27.75 \%$ of the area in Nansebo forest. Quantitative Biodiversity indices such as $\mathrm{H}^{\prime}, \mathrm{E}, \gamma$ and $\alpha$ to measure species richness were used to calculate the bird species diversity and two wayANOVA was used to test for significant variation in species richness and abundance per season among habitats. A total of 105 bird species consisting of 1endemic, 8 near endemics, 1 globally threatened and 9 Palearctic migrants were recorded in Nansebo forest. Species richness and abundance varied between the three habitats in Nansebo forest. There was significant difference in species richness among habitats in Nansebo forest (F1, 18=94.657 $\mathrm{P}=0.000)$ at 0.001 significance level. In Nansebo forest the highest Shannon-Wiener diversity index $\left(\mathrm{H}^{\prime}=4.17\right)$ was recorded from the modified habitat. It can be concluded that the area has high species diversity including endemics and endangered species. It can also be good potential for bird watching tourism that can integrate economic gain with biodiversity conservation. Hence, urgent conservation measures and further detail research is recommended to conserve the bird species.
\end{abstract}

Keywords/Phrases: Avian species, Habitat types, Species richness, Species similarity

DOI: $10.7176 / \mathrm{JBAH} / 9-5-04$

Publication date:March $31^{\text {st }} 2019$

\section{Introduction}

Ethiopia has rich biodiversity resources of which 926 bird species comprising of 24 endemics (EBI, 2015 and Weldemariam Tesfahunegny, 2016). Birds are one of the most important components of biodiversity with ecological, economic and esthetic values. Birds are known as efficient and cost-effective insect pest controllers, Fruit-eating birds help in seed dispersal and seeds may grow everywhere in the fall of droppings and certain birds like hummingbirds and sunbirds also used for flower pollination that produce nectar. They are also the source of considerable fascination and folklore and have been used as symbols (Clout and Hay, 1989).

The distribution and abundance of many bird species are determined by the composition of the vegetation or habitat (Lee and Rotenberry, 2005). Climate influences habitats and movements of resident and migratory birds that are characteristic of particular habitats or biomes (Metzger et al., 2009). Studies from different parts of the world focused on characterizing the bird's species composition and abundance on specific regions of the country (eg. Study by Girma and Afework, 2008). There are a few isolated reports of bird species diversity outside of protected areas in Ethiopia (Aerts et al., 2008), although there is better documentation of birds in protected areas (EWNHS, 1996). Comprehensive baseline information is lacking even for several of the endemic bird species. The status of birds in the present study areas is very little known. As a result, the present study is aimed to investigate species diversity and relative abundance of birds in nansebo forest and contribute to the conservation of the species in the area.

\section{Materials and Methods}

Study area

The study was conducted in Nansebo forest that found in Nansebo District in west Arsi Zone of Oromiya Regional state of Ethiopia (Fig.1). Nansebo district is located between $6^{\circ} 10^{\prime}-6^{\circ} 40^{\prime} \mathrm{N}$ and $39^{\circ} 0^{\prime}-39^{\circ} 40^{\prime} \mathrm{E}$ (Fig.1). It is located $407 \mathrm{~km}$ far from Addis Ababa and $134.5 \mathrm{~km}$ away from Shashemene, the capital city of the zone. 


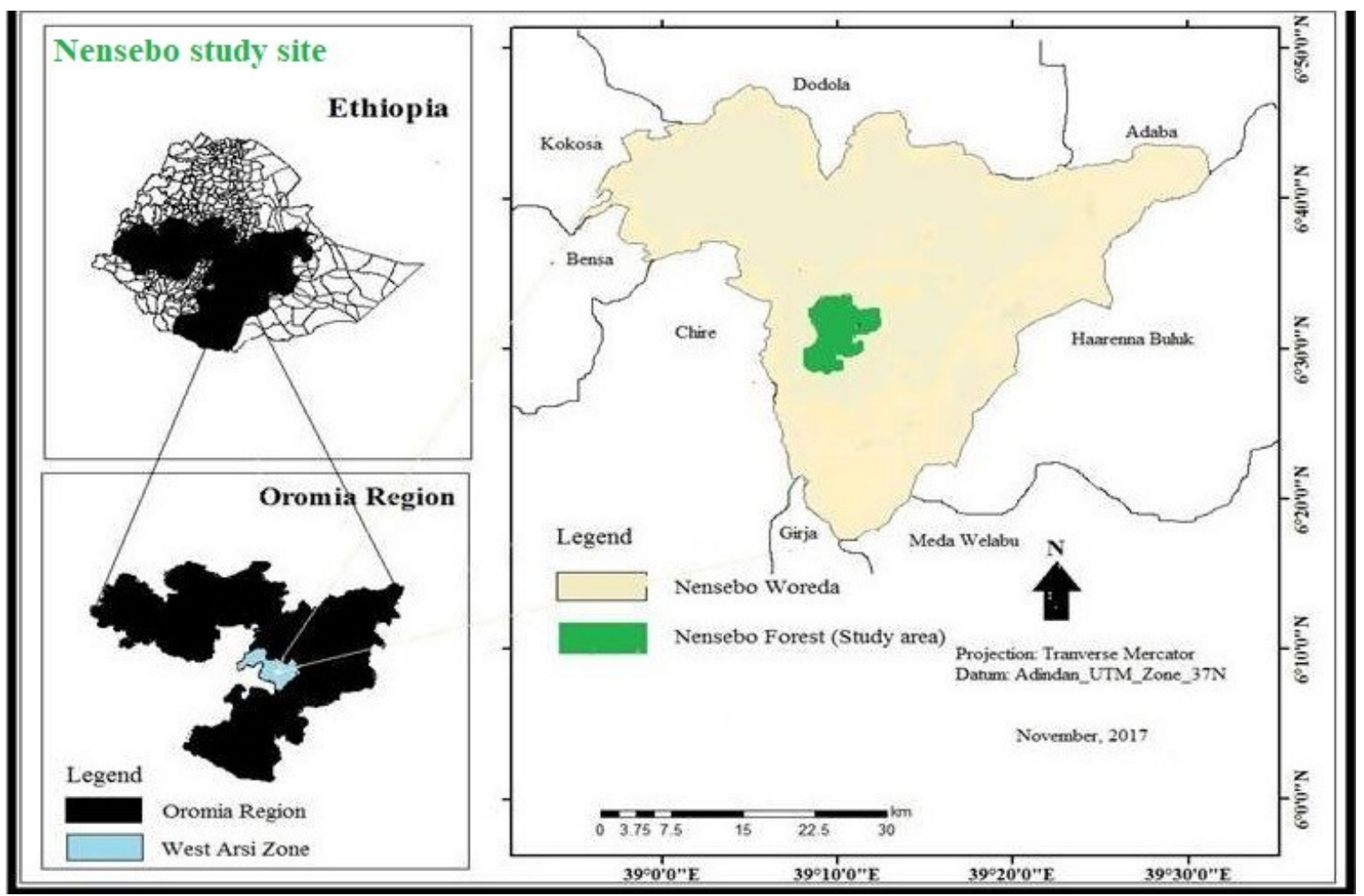

Figure 1: Location Map of Study Area

Nansebo district is characterized by mountainous landscape having an altitude ranges from $1500 \mathrm{~m}$ a.s.l to 3700 $\mathrm{m}$ a.s.l. The district exhibit bimodal rainfall pattern, with the annual rainfall ranges between 900 to $1100 \mathrm{~mm}$ with a temperature that varies between a minimum of $15 \mathrm{C}^{\circ}$ and a maximum $22 \mathrm{C}^{\circ}(\mathrm{NWAO}, 2012)$.

\section{Stratification of the study area and sampling design}

For this study, Nansebo forest was stratified into two habitat types; modified habitat at the lower and Moist Afromontane forest at the higher altitude following vegetation type and altitudinal gradient. In Nansebo forest, modified habitat represents areas with altitudes occurs from 1882-2153m a.s.l. Moist Afromontane forest represents altitude areas between $2186-2392 \mathrm{~m}$ a.s.l, with dominant stands of the indigenous tree species such as Croton macrostachys, Strychnos spinosa, Clematis longicauda, Prunus africana and Millettia ferruginea. The Moist Afromontane forest was relatively intact and undisturbed compared with modified habitat.

Based on the reconnaissance survey, sampling transects was systematically generated in a geographic information system (GIS) using ArcGIS software v. 10.1 (ESRI, 2012) in the Nansebo forest. Line transect method was used since the study area is accessible and species can be detected along transect line. With line transect method it is possible to cover large areas and can generate more species richness efficiently (Bibby et al., 1992). Therefore, this method is very important since comprehensive baseline information and status of bird species in Nansebo forest is lacking.

The total area of Nansebo forest was 11,350 ha. Of these $27.75 \%$ (3150 ha) of the area was sampled in Nansebo forest. A stratified random sampling technique was employed in which transect placement was proportional to the area of the habitat types and represents each of the habitat types (Bibby et al., 1998, Shimelis and Afework, 2008).

Accordingly, a total 20 transect lines, of which six transects in modified habitat while the rest fifteen (15) transects were used in Moist Afromontane forest to estimate the species diversity and relative abundance of birds in Nansebo forest (Fig. 2).

The distance between two adjacent transects was $1 \mathrm{~km}$ and the length of each transect line was $1 \mathrm{~km}$ with a width of $0.25 \mathrm{~km}$ or less in Nansebo forest. To avoid edge effect, transect lines were spaced $500 \mathrm{~m}$ from the roadside (edge of the forest). 


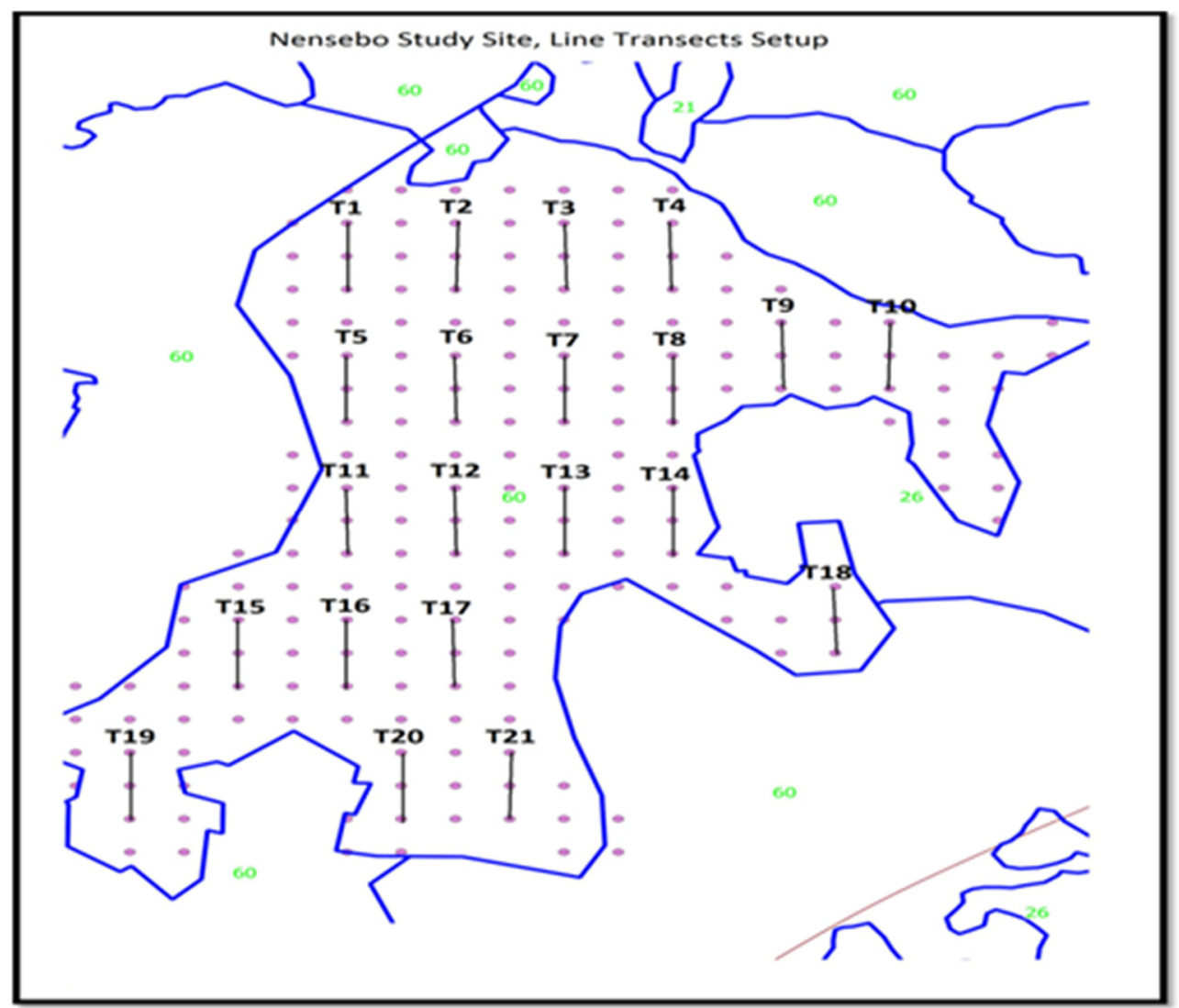

Figure 2: Line transect layout of the Nansebo forest

\section{Data collection and analysis}

Data collection was carried out on foot walking along transect lines. Bird identifications and counting of individuals conducted by direct observations aided with naked eye and binoculars (10x50). Sound records and photography were also taken for further confirmation by using Digital Camera. Location and distance of the observed birds was determined and recorded along transect lines using Geographic positioning system (GPS). Each day of survey, arrive at the starting point approximately 20 minutes before sunrise so that counting can begin at sunrise to minimize the effect of time and weather conditions on bird detectability.

Birds were counted when they were active in the mornings from 06:30-10:00 $\mathrm{h}$ and in the afternoon from 15:30-18.00 h (Bibby et al., 1992; Centerbury et al., 2000). Unfavorable weather (strong wind or rain) was also being considered. A bird flying over the area was observed and recorded on data gathering worksheets to identify for species richness. For identification of species, plumage pattern, size, shape, color, songs and calls were considered as important parameters (Afework and Shimelis, 2009). Songs and calls were also used for identifying nocturnal species.

Avian diversity of each habitat analyzed using Shannon-Wiener diversity Index (H') (Shannon and Wiener, 1949). Quantitative indices to measure species richness was used as $\alpha$-diversity which is the average species richness per transect within a given habitat and $\gamma$-diversity as the habitat level richness (Schmitt et al., 2010).

Percent relative abundance was calculated using formula $(\%)=\mathrm{n} / \mathrm{N} \times 100$ where, $\mathrm{n}$ is the number of individuals of particular species recorded and $\mathrm{N}$ is the total number of individuals of the species. Data obtained during the survey was analyzed by using two-way ANOVA to analyze the effect of season and habitat on bird species richness and abundance.

\section{RESULTS}

\section{Diversity}

In Nansebo forest variation in the number of bird species was observed among the habitats and between seasons in the same habitat. The highest mean number of species per transect was recorded from modified habitat during wet $(10.8 \pm 3.21)$ and dry $(8.6 \pm 2.25)$ seasons (Table 1). The Moist Afromontane forest accounts mean species richness (5.5 \pm 2.47$)$ during wet season and (5.2 \pm 1.02$)$ during dry season in Nansebo forest (Table 1). The evidence from the present study shows variations were also observed in species diversity among the different habitat types during the wet and dry seasons in Nansebo forest. The highest avian diversity was recorded from modified habitat during dry $\left(H^{\prime}=4.17\right)$ and wet $\left(H^{\prime}=3.99\right)$ seasons $($ Table 1$)$. 
The Modified habitat had the evenness of bird species recorded during dry season $(E=0.957$ and wet $(E=0.910)$ (Table 1).

Table 1: Seasonal variation in bird diversity in Nansebo Forest among the different habitat types

\begin{tabular}{|c|c|c|c|c|c|c|c|c|c|}
\hline \multirow[b]{2}{*}{$\begin{array}{l}\text { Study } \\
\text { areas }\end{array}$} & \multirow[b]{2}{*}{ Habitat types } & \multicolumn{8}{|c|}{ Diversity Measures } \\
\hline & & Season & $\begin{array}{l}\text { BSRPH }(\gamma \\
\text {-diversity })\end{array}$ & $\begin{array}{c}\text { BMSRPT( } \alpha- \\
\text { diversity) }\end{array}$ & BSAPH & BMSAPT & H' & $\mathrm{H}^{\prime} \max$ & Evenness \\
\hline \multirow{4}{*}{$\begin{array}{l}\text { Nansebo } \\
\text { Forest } \\
\end{array}$} & Moist & dry & 78.000 & $5.2 \pm 1.02$ & 221.000 & $14.73 \pm 2.437$ & 3.620 & 3.761 & 0.963 \\
\hline & $\begin{array}{l}\text { Afromontane } \\
\text { forest }\end{array}$ & wet & 82.000 & $5.5 \pm 2.47$ & 554.000 & $36.93 \pm 1.931$ & 3.670 & 3.989 & 0.920 \\
\hline & \multirow{2}{*}{ Modified } & dry & 43.000 & $8.6 \pm 2.25$ & 107.000 & $21.4 \pm 3.873$ & 4.170 & 4.357 & 0.957 \\
\hline & & wet & 54.000 & $10.8 \pm 3.21$ & 218.000 & $43.6 \pm 3.873$ & 3.990 & 4.407 & 0.910 \\
\hline
\end{tabular}

BSRPH: Bird species richness per habitat

BMSRPT: Bird mean species richness per transect

BSAPH: Bird species abundance per habitat

BMSAPT: Bird mean species abundance per transect

In Nansebo forest in terms of habitat types, variation in the number of bird species was observed among the habitats. The highest number of average species richness $(4.70 \pm 1.647)$ and individuals $(32 \pm 4.103)$ was recorded from modified habitat followed by Moist Afromontane forest in average species richness $(3.95 \pm 4.128)$ and individuals $(25 \pm 7.535)$ in Nansebo forest. There was significant difference in mean species richness among habitats $(\mathrm{F} 1,18=94.657 \mathrm{P}=0.000)$, and insignificant in species abundance $(\mathrm{F} 1,18=0.853 \mathrm{P}=0.368)$ at 0.05 significance level in Nansebo forest (Appendix 2).

The highest bird diversity $\left(\mathrm{H}^{\prime}=4.131\right)$ was also recorded from modified habitat. The highest even distribution $(\mathrm{E}=0.956)$ was observed in Moist Afromontane forest (Table 2).

Table 2: Bird diversity in Nansebo forest among habitat types

\begin{tabular}{|c|c|c|c|c|c|c|c|c|}
\hline \multirow[b]{2}{*}{$\begin{array}{l}\text { Study } \\
\text { areas }\end{array}$} & \multirow[b]{2}{*}{ Habitat types } & \multicolumn{7}{|c|}{ Diversity Measures } \\
\hline & & $\begin{array}{l}\text { SRPH }(\gamma- \\
\text { diversity) }\end{array}$ & $\begin{array}{c}\text { MSRPT( } \alpha- \\
\text { diversity) }\end{array}$ & $\begin{array}{c}\text { SAP } \\
\text { H }\end{array}$ & $\begin{array}{c}\text { MSA } \\
\text { PT }\end{array}$ & $\mathbf{H}^{\prime}$ & $\begin{array}{c}\mathbf{H}^{\prime} \\
\max \end{array}$ & $\begin{array}{c}\text { Evenn } \\
\text { ess }\end{array}$ \\
\hline & Moist & & & & & & & \\
\hline & Afromontane & 06 & $305+4128$ & 764 & $25 \pm 7$. & $\begin{array}{l}3.7 \\
05\end{array}$ & 3070 & 0056 \\
\hline Nansebo & IOIESt & 90 & & & $32 \pm 4$ & $\begin{array}{l}95 \\
4.1\end{array}$ & 3.970 & 0.950 \\
\hline Forest & Modified & 53 & $4.70 \pm 1.647$ & 163 & 103 & 31 & 4.564 & 0.905 \\
\hline
\end{tabular}

SRPH: Species richness per habitat

MSRPT: Mean species richness per transect

SAPH: Species abundance per habitat

MSAPT: Mean species abundance per transect

\section{Species Accumulation Curve}

The species accumulation curve of bird species of Nansebo forest flattened before the total numbers of samples considered were exhausted. The following species accumulation curve showing that sufficient numbers of samples were considered to determine bird species diversity of each habitat (Figure 3: A and B).
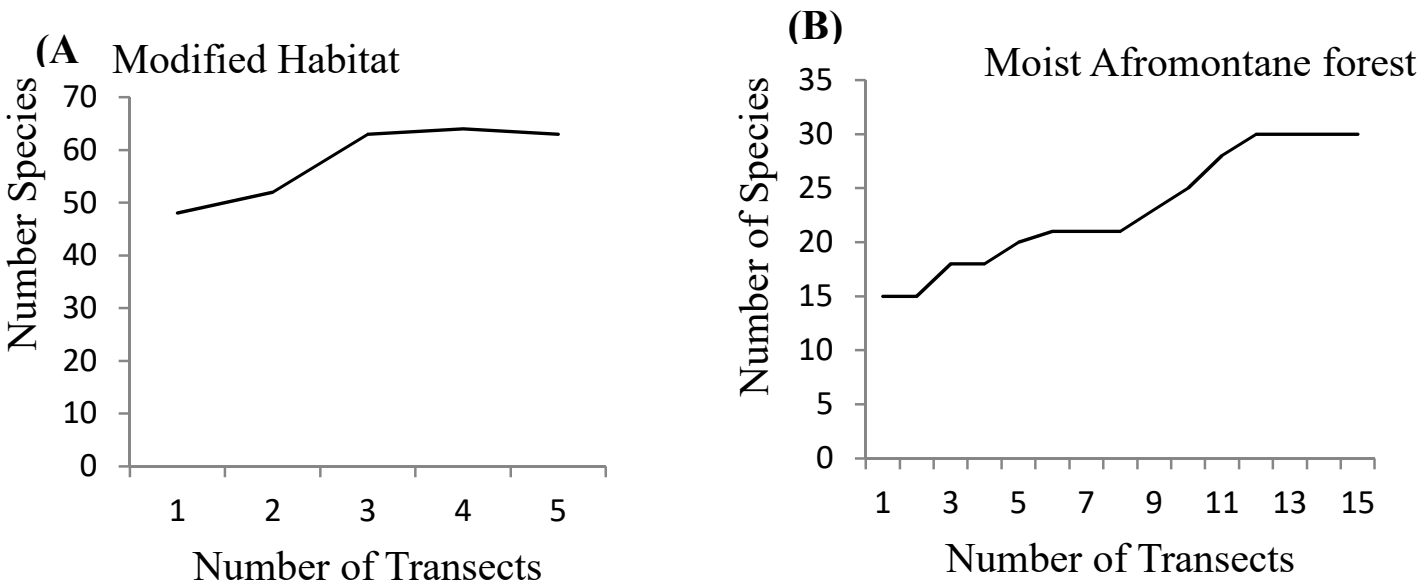

Figure 3 Aand B: Species Accumulation curve of Nansebo forest per habitat types. 


\section{Species Composition and Relative Abundance}

A total of 105 species were recorded from Nansebo forests (Table 4). Among the recorded species, Wattled ibis (Bostrychia carunculata), Thick billed raven (Corvus crassirostris), Black winged love bird (Agapornis taranta), Ethiopian cisticola (Cisticola lugubris), Abyssinian oriole (oriolus monacha), Abyssinian slaty flycatcher (Chocolatina ficedula), Abyssinian wood pecker (Abyssinicus phylloscopus) and Banded Barbet (Lybius undatus) were endemic to Ethiopia and Eritrea in Nansebo forest. The endemic Yellow-fronted Parrot (Poicephalus flavifrons) was also recorded from Nansebo forest.

One globally threatened bird species i.e Semi collared flycatcher (Semi torquata) were near threatened bird species (IUCN red list, 2016) (Table 3).

Among the recorded bird species, 96 species were resident whereas 9 were Palearctic migrants' species (Table $3)$.

In Nansebo forest the highest number of bird species was recorded from the family Sylviidae (10 species) followed by Accipitridae ( 8 species), Columbidae ( 7 species) and Cisticolidae (6 species) (Table 3 ).

Table 3: Bird species recorded at Nansebo forest (a, Near Endemic c, endangered b, Endemic e, near threatened NM, Northern Migratory $\quad$ AM, Inter-African migrant)

\begin{tabular}{|c|c|c|c|c|c|c|c|c|}
\hline \multirow[t]{2}{*}{ Order } & \multirow[t]{2}{*}{ Family } & \multirow[t]{2}{*}{ Common Name } & \multirow[t]{2}{*}{ Scientific Name } & \multicolumn{3}{|c|}{ Abundance } & \multirow[b]{2}{*}{ RA (\%) } & \multirow[b]{2}{*}{ Rank } \\
\hline & & & & Wet & Dry & Total & & \\
\hline Passeriformes & Oriolidae & Abyssinian oriole & Oriolus monach $a^{\mathbf{a}}$ & 68 & 46 & 74 & 6.9 & $1^{\mathrm{st}}$ \\
\hline Passeriformes & Turdidae & Mountain thrush & Turdus olivaceus & 34 & 14 & 48 & 4.5 & $2^{\text {nd }}$ \\
\hline Passeriformes & Zosteropidae & Montane white eye & Zosterops poliogastrus & 23 & 12 & 35 & 3.3 & $3^{\text {rd }}$ \\
\hline Passeriformes & Passeridae & Swaisons sparrow weaver & Passer swainsonii & 22 & 11 & 33 & 3.1 & $4^{\text {th }}$ \\
\hline Passeriformes & 'Sturnidae & Red winged starling & Onychognathus morio & 17 & 13 & 30 & 2.8 & $5^{\text {th }}$ \\
\hline Passeriformes & Fringillidae & Streaky seed eater & Serinus striolatus & 22 & 7 & 29 & 2.7 & $6^{\text {th }}$ \\
\hline Passeriformes & Estrildidae & Yellow bellied waxbill & Coccopygia quartinia & 19 & 10 & 29 & 2.7 & $6^{\text {th }}$ \\
\hline Passeriformes & ploceidae & Baglafecht weaver & Ploceus baglafecht & 16 & 7 & 26 & 2.4 & $8^{\text {th }}$ \\
\hline Passeriformes & Oriolidae & Black headed oriole & Oriolus larvatus & 13 & 3 & 26 & 2.4 & $8^{\text {th }}$ \\
\hline Passeriformes & Muscicapidae & Abyssinian slaty flycatcher & Melaenornis chocolatin $a^{\mathbf{a}}$ & 16 & 9 & 25 & 2.3 & $10^{\text {th }}$ \\
\hline Passeriformes & Muscicapidae & Semi collared flycatcher & Ficedula semitorquata $^{\mathbf{e}}$ & 22 & 3 & 25 & 2.3 & $10^{\text {th }}$ \\
\hline Passeriformes & Nectariniidae & Variable sun bird & Cinnyris venustus & 16 & 7 & 23 & 2.1 & $12^{\text {th }}$ \\
\hline Passeriformes & 'Pycnonotidae & Common bulbul & Pycnonotus barbatus & 12 & 10 & 22 & 2.1 & $12^{\text {th }}$ \\
\hline Trogoniformes & Trogonidae & Narina's Trogon & Apaloderma narina & 15 & 5 & 20 & 1.9 & $14^{\text {th }}$ \\
\hline Passeriformes & Viduidae & Pin tailed whydah & Vidua macroura & 16 & 4 & 20 & 1.9 & $14^{\text {th }}$ \\
\hline Passeriformes & Muscicapidae & Rupels robin chat & Cossypha semirufa & 11 & 9 & 20 & 1.9 & $14^{\text {th }}$ \\
\hline Passeriformes & Corvidae & Thick billed raven & Corvus crassirostris $^{\mathbf{a}}$ & 12 & 7 & 19 & 1.8 & $17^{\text {th }}$ \\
\hline Piciformes & Indicatoridae & Greater honey guide & Indicator indicator & 16 & 2 & 18 & 1.7 & $17^{\text {th }}$ \\
\hline Passeriformes & Estrildidae & Red billed fire finch & Lagonosticta senegala & 14 & 4 & 18 & 1.7 & $17^{\text {th }}$ \\
\hline Passeriformes & Monarchidae & African dusky flycatcher & Muscicapa adusta & 9 & 7 & 16 & 1.5 & $20^{\text {th }}$ \\
\hline Passeriformes & Sylviidae & Cinnamon bracken warbler & Bradypterus cinnamomeus & 14 & 2 & 16 & 1.5 & $20^{\text {th }}$ \\
\hline Passeriformes & Fringillidae & African citril & Serinus citrinelloides & 9 & 6 & 15 & 1.4 & $22^{\text {th }}$ \\
\hline Galliformes & phasianidae & Chestnut naped francolin & Pternistis castaneicollis & 11 & 4 & 15 & 1.4 & $22^{\text {th }}$ \\
\hline Passeriformes & Cisticolidae & Ethiopian cisticola & Cisticola lugubris ${ }^{\mathbf{a}}$ & 13 & 2 & 15 & 1.4 & $22^{\text {th }}$ \\
\hline Passeriformes & Cisticolidae & Green backed cameroptera & Camaroptera brachyura & 6 & 9 & 15 & 1.4 & $22^{\text {th }}$ \\
\hline Psittaciformes & Psittaculidae & Black winged love bird & Agapornis taranta ${ }^{\mathbf{a}}$ & 10 & 4 & 14 & 1.3 & $26^{\text {th }}$ \\
\hline Psittaciformes & Nectariniidae & Collared sun bird & Hedydipna collaris & 12 & 2 & 14 & 1.3 & $26^{\text {th }}$ \\
\hline Columbiformes & Columbidae & Red eyed dove & Strepetopelia semitorquata & 10 & 4 & 14 & 1.3 & $26^{\text {th }}$ \\
\hline Passeriformes & Monarchidae & *African paradise flycatcher ${ }^{\mathbf{A M}}$ & Terpsiphone viridis & 9 & 4 & 13 & 1.2 & $29^{\text {th }}$ \\
\hline Passeriformes & Fringillidae & Brown rumped seed eater & Crithagra tristriatus & 8 & 5 & 13 & 1.2 & $29^{\text {th }}$ \\
\hline Piciformes & Indicatoridae & Lesser honeyguide & Indicator minor & 9 & 4 & 13 & 1.2 & $29^{\text {th }}$ \\
\hline Accipitriformes & Accipitridae & Lesser spotted eagle & Aquila pomarina & 8 & 4 & 12 & 1.1 & $32^{\text {th }}$ \\
\hline Cuculiformes & Cuculidae & ${ }^{*}$ Levaillant's cuckoo ${ }^{\mathbf{A M}}$ & Clamator levaillantii & 9 & 2 & 11 & 1.0 & $33^{\text {th }}$ \\
\hline Passeriformes & Fringillidae & Yellow crowned canary & Crithagra mozambicus & 11 & 0 & 11 & 1.0 & $33^{\text {th }}$ \\
\hline Passeriformes & Cisticolidae & Tawny flanked prinia & Prinia subflava & 6 & 4 & 10 & 0.9 & $35^{\text {th }}$ \\
\hline Cuculiformes & Musophagidae & White cheeked turaco & Tauraco leucotis & 4 & 6 & 10 & 0.9 & $35^{\text {th }}$ \\
\hline Piciformes & Picidae & Abyssinian wood pecker & Dendropicos abyssinicus & 4 & 5 & 9 & 0.8 & $37^{\text {th }}$ \\
\hline Passeriformes & Sylviidae & Brown wood warbler & Phylloscopusumbrovirens ${ }^{\mathbf{a}}$ & 7 & 2 & 9 & 0.8 & $37^{\text {th }}$ \\
\hline Passeriformes & Laniidae & Common fiscal & Lanius collaris & 7 & 2 & 9 & 0.8 & $37^{\text {th }}$ \\
\hline Coliiformes & Collidae & Speckled mouse bird & Colius striatus & 5 & 4 & 9 & 0.8 & $37^{\text {th }}$ \\
\hline Psittaciformes & Psittacidae & Yellow fronted parrot & Poicephalusflavifrons ${ }^{\mathbf{b}}$ & 7 & 2 & 9 & 0.8 & $37^{\text {th }}$ \\
\hline Passeriformes & Passeridae & Bush petronia & Petronia dentata & 6 & 2 & 8 & 0.7 & $42^{\text {th }}$ \\
\hline Columbiformes & Columbidae & Dusky turtle dove & Streptope lialugens & 2 & 6 & 8 & 0.7 & $42^{\text {th }}$ \\
\hline Passeriformes & Buphagidae & Red billed oxpecker & Buphagus erythrorhynchus & 4 & 4 & 8 & 0.7 & $42^{\text {th }}$ \\
\hline Piciformes & Indicatoridae & Scaly throated honey guide & Indicator variegatus & 8 & 0 & 8 & 0.7 & $42^{\text {th }}$ \\
\hline Coraciiformes & Alcedinidae & Wood land kingfisher & Halcon senegalensis & 3 & 5 & 8 & 0.7 & $42^{\text {th }}$ \\
\hline Passeriformes & Sylviidae & Wood warbler & Phylloscopus sibilatrix & 6 & 2 & 8 & 0.7 & $42^{\text {th }}$ \\
\hline Falconiformes & Accipitridae & Augur buzzard & Buteo augur & 4 & 3 & 7 & 0.7 & $42^{\text {th }}$ \\
\hline Cuculiformes & Sylviidae & Brown parisoma & Parisoma lugens & 5 & 2 & 7 & 0.7 & $42^{\text {th }}$ \\
\hline Piciformes & Picidae & Eastern grey wood pecker & Dendropicos goertae & 5 & 2 & 7 & 0.7 & $42^{\text {th }}$ \\
\hline Piciformes & Picidae & Nubian wood pecker & Campethera nubica & 5 & 2 & 7 & 0.7 & $42^{\text {th }}$ \\
\hline Columbiforme & Columbidae & ${ }^{*}$ Tambourine dove ${ }^{\mathrm{AM}}$ & Turtur tympanistria & 6 & 1 & 7 & 0.7 & $42^{\text {th }}$ \\
\hline Cuculiformes & Cuculidae & African emerald cuckoo & Chrysococcyx cupreus & 4 & 2 & 6 & 0.6 & $53^{\text {th }}$ \\
\hline Piciformes & Lybiidae & Banded barbet & Lybius undatus ${ }^{\mathbf{a}}$ & 3 & 3 & 6 & 0.6 & $53^{\text {th }}$ \\
\hline Passeriformes & Hirundinidae & *Barn swallow ${ }^{\mathrm{NM}}$ & Hirundo rustica & 2 & 4 & 6 & 0.6 & $53^{\text {th }}$ \\
\hline Passeriformes & Sylviidae & Black start & Cercomela melanura & 6 & 0 & 6 & 0.6 & $53^{\text {th }}$ \\
\hline Passeriformes & Estrildidae & Common waxbill & Estrilda astrild & 6 & 0 & 6 & 0.6 & $53^{\text {th }}$ \\
\hline Passeriformes & Buphagidae & Grey cuckoo shrike & Coranica caesia & 4 & 2 & 6 & 0.6 & $53^{\text {th }}$ \\
\hline Passeriformes & Turdidae & Ground scraper thrush & Psophocichla litsipsirupa & 4 & 2 & 6 & 0.6 & $53^{\text {th }}$ \\
\hline Passeriformes & Sylviidae & Little rush warbler & Bradypterus baboecala & 4 & 2 & 6 & 0.6 & $53^{\text {th }}$ \\
\hline Passeriformes & Muscicapidae & Northern black flycatcher & Melaenornis edolioides & 6 & 0 & 6 & 0.6 & $53^{\text {th }}$ \\
\hline
\end{tabular}




\begin{tabular}{|c|c|c|c|c|c|c|c|c|}
\hline \multirow[t]{2}{*}{ Order } & \multirow[t]{2}{*}{ Family } & \multirow[t]{2}{*}{ Common Name } & \multirow[t]{2}{*}{ Scientific Name } & \multicolumn{3}{|c|}{ Abundance } & \multirow[b]{2}{*}{ RA (\%) } & \multirow[b]{2}{*}{ Rank } \\
\hline & & & & Wet & Dry & Total & & \\
\hline Coraciiformes & Coraciidae & Abyssinian roller & Coracias abyssinicus & 5 & 0 & 5 & 0.5 & $53^{\text {th }}$ \\
\hline Apodiformes & Apodidae & African black swift & Apus barbatus & 3 & 2 & 5 & 0.5 & $53^{\text {th }}$ \\
\hline Columbiformes & Columbidae & Black billed wood dove & Turtur abyssinicus & 4 & 1 & 5 & 0.5 & $53^{\text {th }}$ \\
\hline Passeriformes & Buphagidae & Black cuckoo shrike & Campephaga flava & 3 & 2 & 5 & 0.5 & $53^{\text {th }}$ \\
\hline Accipitriformes & Buphagidae & Lesser white throat & Sylvia curruca & 5 & 0 & 5 & 0.5 & $53^{\text {th }}$ \\
\hline Cuculiformes & Cuculidae & Red chested cuckoo & Cuculus solitarius & 5 & 0 & 5 & 0.5 & $53^{\text {th }}$ \\
\hline Passeriformes & Cisticolidae & Red faced cisticola & Cisticola erythrops & 3 & 2 & 5 & 0.5 & $53^{\text {th }}$ \\
\hline Ciconiiformes & Threskiornithidae & Silvery checked hornbill & Bycanistes brevis & 3 & 5 & 5 & 0.5 & $53^{\text {th }}$ \\
\hline Cuculiformes & Paridae & White winged black tit & Parus leucomelas & 4 & 1 & 5 & 0.5 & $53^{\text {th }}$ \\
\hline Accipitriformes & Cisticolidae & Yellow breasted apalis & Apalis flavida & 5 & 0 & 5 & 0.5 & $53^{\text {th }}$ \\
\hline Pelecaniformes & Timaliidae & Abyssinian cat bird & Parophasma galinieri ${ }^{\mathbf{b}}$ & 3 & 1 & 4 & 0.4 & $72^{\text {th }}$ \\
\hline Piciformes & Lybiidae & Black billed barbet & Lybius guifsobalito & 4 & 0 & 4 & 0.4 & $72^{\text {th }}$ \\
\hline Columbiformes & Columbidae & Ring necked dove & Streptopelia capicola & 2 & 2 & 4 & 0.4 & $72^{\text {th }}$ \\
\hline Accipitriformes & Accipitridae & Rupels vulture & Gyps rueppellii & 0 & 4 & 4 & 0.4 & $72^{\text {th }}$ \\
\hline Falconiformes & Accipitridae & *Tawny eagle ${ }^{\mathrm{NM}}$ & Aquila rapax & 3 & 1 & 4 & 0.4 & $72^{\text {th }}$ \\
\hline Pelecaniformes & Threskiornithidae & Wattled ibis & Bostrychia carunculata & 2 & 2 & 4 & 0.4 & $72^{\text {th }}$ \\
\hline Falconiformes & Accipitridae & White backed vulture & Gyps africanus & 4 & 0 & 4 & 0.4 & $72^{\text {th }}$ \\
\hline Passeriformes & Sylviidae & Willow warbler & Phylloscopus trochilus & 0 & 4 & 4 & 0.4 & $72^{\text {th }}$ \\
\hline Passeriformes & Cisticolidae & Buff-bellied warbler & Phyllolais pulchella & 0 & 3 & 3 & 0.3 & $80^{\text {th }}$ \\
\hline Passeriformes & Sylviidae & Dark caped yellow warbler & Chloropeta natalensis & 2 & 1 & 3 & 0.3 & $80^{\text {th }}$ \\
\hline Passeriformes & Motacillidae & *Yellow wagtail ${ }^{\mathrm{NM}}$ & Motacilla flava & 3 & 0 & 3 & 0.3 & $80^{\text {th }}$ \\
\hline Passeriformes & Estrildidae & African fire finch & Lagonosticta rubricata & 0 & 2 & 2 & 0.2 & $81^{\text {th }}$ \\
\hline Columbiformes & Columbidae & African olive pegion & Columba arquatrix & 0 & 2 & 2 & 0.2 & $81^{\text {th }}$ \\
\hline Coraciiformes & Alcedinidae & African pigmy kingfisher & Cevx pictus & 2 & 0 & 2 & 0.2 & $81^{\text {th }}$ \\
\hline Passeriformes & Sylviidae & *Black cap ${ }^{\mathrm{NM}}$ & Sylvia atricapilla & 0 & 2 & 2 & 0.2 & $81^{\text {th }}$ \\
\hline Piciformes & Platysteiridae & Black headed batis & Batis minor & 0 & 2 & 2 & 0.2 & $81^{\text {th }}$ \\
\hline Columbiformes & phoeniculidae & Black- billed wood hoopoe & Phoeniculus somaliensis & 2 & 0 & 2 & 0.2 & $81^{\text {th }}$ \\
\hline Cuculiformes & Cuculidae & Blue headed coucal & Centropus monachus & 0 & 2 & 2 & 0.2 & $81^{\text {th }}$ \\
\hline Passeriformes & Sylviidae & ${ }^{*}$ Common redstart ${ }^{\mathrm{NM}}$ & Phoeniculus phoenicurus & 2 & 0 & 2 & 0.2 & $81^{\text {th }}$ \\
\hline Coraciiformes & Bucerotidae & Crowned hornbill & Tockus alboterminatus & 2 & 0 & 2 & 0.2 & $81^{\text {th }}$ \\
\hline Columbiformes & Threskiornithidae & Hadada ibis & Bostrychia hagedash & 2 & 0 & 2 & 0.2 & $81^{\text {th }}$ \\
\hline Passeriformes & 'Malaconotidae & Northern puff back & Dryoscopus gambensis & 0 & 2 & 2 & 0.2 & $81^{\text {th }}$ \\
\hline Passeriformes & Nectariniidae & Takazze sun bird & Nectarinia tacazze & 0 & 2 & 2 & 0.2 & $81^{\text {th }}$ \\
\hline Ciconiiformes & Ciconiidae & Woolly-necked stork & Ciconia episcopus & 0 & 2 & 2 & 0.2 & $81^{\text {th }}$ \\
\hline Passeriformes & Turdidae & Abyssinian ground thrush & Zoothera piaggiae & 0 & 1 & 1 & 0.1 & $96^{\text {th }}$ \\
\hline Falconiformes & strigidae & Cape eagle owl & Bubo capensis & 0 & 1 & 1 & 0.1 & $96^{\text {th }}$ \\
\hline Passeriformes & Corvidae & Fan tailed raven & Corvus rhipidurus & 1 & 0 & 1 & 0.1 & $96^{\text {th }}$ \\
\hline Falconiformes & strigidae & Greyish eagle owl & Bubo cinerascens & 1 & 0 & 1 & 0.1 & $96^{\text {th }}$ \\
\hline Pelecaniformes & Ardeidae & Little egret & Egretta garzetta & 0 & 1 & 1 & 0.1 & $96^{\text {th }}$ \\
\hline Accipitriformes & Accipitridae & Lizzard buzzard & Kaupifalco monogrammicus & 0 & 1 & 1 & 0.1 & $96^{\text {th }}$ \\
\hline Passeriformes & Muscicapidae & Mocking cliff chat & Thamnolaea cinnamomeiventris & 1 & 0 & 1 & 0.1 & $96^{\text {th }}$ \\
\hline Columbiformes & Columbidae & Namaqua dove & Oena capensis & 0 & 1 & 1 & 0.1 & $96^{\text {th }}$ \\
\hline Accipitriformes & Accipitridae & Pallied harrier ${ }^{\mathrm{NM}}$ & Circus macrourus & 0 & 1 & 1 & 0.1 & $96^{\text {th }}$ \\
\hline Accipitriformes & Accipitridae & Yellow billed kite & Milvus(migrans) aegyptius & 0 & 1 & 1 & 0.1 & $96^{\text {th }}$ \\
\hline
\end{tabular}

Based on the percent relative abundance computation, Abyssinian oriole (Oriolus monacha) (6.92\%) was the most abundant bird species when all habitat types considered together in Nansebo forest (3).

When each habitat type was considered separately, Abyssinian oriole (Oriolus monacha) (9.95\%) and Yellow bellied waxbill (Coccopygia quartinia) (5.52\%) were abundant in the Moist Afromontane forest and modified habitat, respectively in Nansebo forest (Table 4).

Table 4: Top five ranking abundant species between two habitat types and across the study area based on percent relative abundance in Nansebo forest

\begin{tabular}{lcccccc}
\hline & \multicolumn{9}{c}{ Habitat Types } \\
\cline { 2 - 7 } & \multicolumn{3}{c}{ Wooded NF } & Modified & & $\begin{array}{c}\text { Across } \\
\text { the study area }\end{array}$ \\
Species Name & RA $(\%)$ & Rank & RA $(\%)$ & Rank & RA (\%) & Rank \\
\hline Abyssinian oriole & 9.95 & $1^{\text {st }}$ & - & - & 6.92 & $1^{\text {st }}$ \\
Mountain thrush & 4.19 & $2^{\text {nd }}$ & 4.91 & $2^{\text {nd }}$ & 4.49 & $2^{\text {nd }}$ \\
Montane white eye & 3.27 & $4^{\text {th }}$ & - & - & 3.27 & $3^{\text {rd }}$ \\
Swaisons sparrow weaver & 3.40 & $3^{\text {rd }}$ & 4.29 & $4^{\text {th }}$ & 3.08 & $4^{\text {th }}$ \\
Red winged starling & 3.01 & $5^{\text {th }}$ & - & - & 2.80 & $5^{\text {th }}$ \\
Yellow bellied waxbill & - & - & 5.52 & $1^{\text {st }}$ & 2.71 & $6^{\text {th }}$ \\
Rupels robin chat & - & - & 4.91 & $3^{\text {rd }}$ & 1.87 & $7^{\text {th }}$ \\
Thick billed raven & - & - & 3.68 & $5^{\text {th }}$ & 1.78 & $8^{\text {th }}$ \\
\hline
\end{tabular}

\section{DISCUSSION}

Diversity

In Nansebo forest the modified habitat had the most diversified avian species and most evenness as compared to the other habitat types. This variation could be due to variation in habitat heterogeneity. The more heterogeneous vegetation and vegetation strata in the modified habitat compared to the other habitat types could have provided several niches for birds making the modified habitat with high diversity of birds. The importance of the availability 
of different vegetation strata for different bird species is also supported by other studies. For example, Cueto and Casenava (1999) reported positive correlation between bird species richness and the availability of vegetation strata. Similarly, Erdelen (1984) indicated significant correlation between bird species diversity and vegetation structure.

The highest number of species recorded from modified habitat during wet and dry seasons. The outcome of this result coincides with the report of Karr (1976); where structurally complex vegetation buffers the influence of seasonality and there is a great stability in resource availability, which allows species to occur as residents throughout the year. Species richness of different feeding guilds might respond differently to changes in vegetation structure and complexity across tropical ecosystems (Oliveira-Filho et al., 2013). During the wet season, flowering plants were flourishing and as a result, food was plentiful for birds in almost all the habitats. However, during the dry season, the deciduous trees defoliate and in the absence of food, many species of birds were restricted to specific habitat where sufficient resource was available.

The variations in species composition recorded during the wet and dry seasons among habitats were significantly different. This is also reported by Aynalem Shimelis and Afework (2008) who showed the important effect of season or the role of climate in affecting the diversity of birds of a particular habitat. According to Karr (1976), the distinct seasonality of rainfall and seasonal variation in the abundance of food resources result in seasonal changes in the abundance of birds.

\section{Relative Abundance}

The relative abundance of bird species during seasons might also be related to the availability of food, habitat condition and breeding season of the species. Therefore, species distribution and abundance can be influenced by seasonal variation. Many factors could account for this. For example, Karr (1976) related the seasonality in the number of bird species with the availability of resources such as food and vegetation strata and found that the number of bird species varied seasonally with peaks in the late dry and early wet seasons.

In Nansebo forest, Abyssinian oriole (Oriolus monacha) had the highest percent relative abundance in Moist Afromontane forest. This was due to the favorable environment of the habitat that supported the species in different ways. Since, Oriolus monacha is a forest specialist species, it could be confined to the natural forest habitat types unlike many other species recorded from the area that tend to concentrate in heterogeneous human modified habitat ignoring the homogenous natural forest. Similar observation has been made in a study carried out in Tanzania, where by forest specialists were only confined to the homogenous forest ignoring the heterogeneous human modified habitat types.

\section{CONCLUSION AND RECOMMENDATIONS}

The Nansebo forest had high bird species diversity including endemic and endangered species revealing the importance of the sites for bird conservation. Therefore, it can serve as good potential for bird watching tourism that can integrate economic gain with biodiversity conservation.

The number of individual species of both study areas shows seasonal variation. This seasonal variation is due to the cumulative effect of both biotic and a biotic factors. The distribution of avian species is also closely related to type of the habitat, which is influenced by environmental factors such as rainfall, altitude, slope, and temperature. The data collected provide valuable information on the ecology of birds and their significance for the environment. Conserving the habitats as well as the species has great biological and social values. Therefore, to maintain the habitat and the avifauna species, the following recommendations are forwarded:

$>$ Conservation work through community participation should be properly developed and practiced.

$>$ As abundance and distribution of the bird species is determined by abundance and distribution of vegetation, equal conservation priority should be given to the bird habitats.

$>$ Further study especially on smaller and cryptic bird species needs to be conducted to provide more information on the diversity of birds in the area.

$>$ Additional detailed studies on bird species together with the other ecological aspects of the birds should be conducted.

\section{Acknowledgement}

We appreciate Wondo Genet College of Forestry and Natural Resources, Hawassa University for funding this research.

\section{REFERENCES}

Aerts, R., F. Lerouge, E. Novmber, L. Lens, M. Hermay and B. Muys. 2008. Land rehabilitation and the conservation of birds in a degraded Afromontane landscape in northern Ethiopia.Biodiversity and Conservation 17: 53-69.

Afework Bekele and Shimelis Aynalem. 2009. Species composition, relative abundance and habitat association 
of the bird fauna of the montane forest of Zegie Peninsula and nearby Islands, Lake Tana, Ethiopia. SINET: Ethiopian Journal of Science 32:45-56.

Bibby, C. J., Burgess, N. D and D. Hills. 1992. Birds census technique. Tokyo, Toronto. Academic press 30: 8696.

Bibby, C.J., S. Marsden, and M. Jones. 1998. Bird surveys. Expedition Advisory Centre.

Clout, M.N. and J.R. Hay. 1989. The importance of birds as browsers, pollinators and seed dispersers in New Zealand forest.New Zealand journal of ecology.27-33.

Cueto, V.R. and J.L. Casenava. 1999. Determinants of bird species richness.Role of climate and vegetation structure at regional scale. Journal of Biogeography 26: 487-492.

Erdelen, M. 1984. Bird communities and vegetation structure: I.Correlations and comparison of simple and diversity indices. Oecologia 61: 277-284.

ESRI . 2012. Arc GIS software 10.1. Environmental Systems Research Institute. California.

EBI. 2015. Ethiopia's national biodiversity strategy and action plan, Addis Ababa ,Ethiopia, 2015-2020.

EWNHS. 1996. Important Bird Areas of Ethiopia. Ethiopian Wildlife Conservation Organization, Addis Ababa, Ethiopia 454p.

Girma Mengesha and Afework Bekele. 2008. Diversity and relative abundance of birds of Alatish National Park. International Journal of Ecology and Environmental Sciences 34:215-222.

Karr, J.R. 1976. Seasonality resource availability and community diversity in tropical bird communities. The American Naturalist 110: 937-974.

Lee, P.Y. and J.T. Rotenberry. 2005. Relationships between bird species and tree species assemblages in forested habitats of north eastern America. Journal of Biogeography 32:1139-1150.

Metzger, J. P., A. C. Martensen, M. Dixo, L. C. Bernacci. M. C.Ribeiro, A. M. G. Teixeira and R. Pardini. 2009. Time-lag in biological responses to landscape changes in a highly dynamic Atlantic forest region. Biological Conservation 142: 1166-1177.

NWAO. 2012. Annual Report. 18-25.

Oliveira-Filho, A. T., J. C. Budke, J. A. Jarenkow, P. V. Eisenlohr, and D. R. M. Neves. 2013. Delving into the variations in tree species composition and richness across South American subtropical Atlantic and Pampean forest. Journal of Plant Ecology 8: 242-260.

Schmitt, C.B., M. Denich, S. Demissew, I. Friis, and H.J. Boehmer, 2010. Floristic diversity in fragmented Afromontane rainforest: Altitudinal variation and conservation importance. Applied Vegetation Science 13: 291-30.

Shannon, C.E. and Wiener, N., 1949. The Mathematical Theory of communication, the University of Illinois, Urbana, p. 117.

Shimelis Aynalem and Afework Bekele. 2008. Species composition, relative abundance and distribution of bird fauna of riverine and wetland habitats of Infranz and Yiganda at Southern tip of Lake Tana, Ethiopia. Tropical Ecology 49: 199-209.

Weldemariam Tesfahunegny Bezabh. 2006. A guide to a complete annotated checklist of the birds of Ethiopia.

\section{APPENDICES}

Appendix 1: ANOVA Table (Species Richness versus Vegetation Height of Nansebo forest )

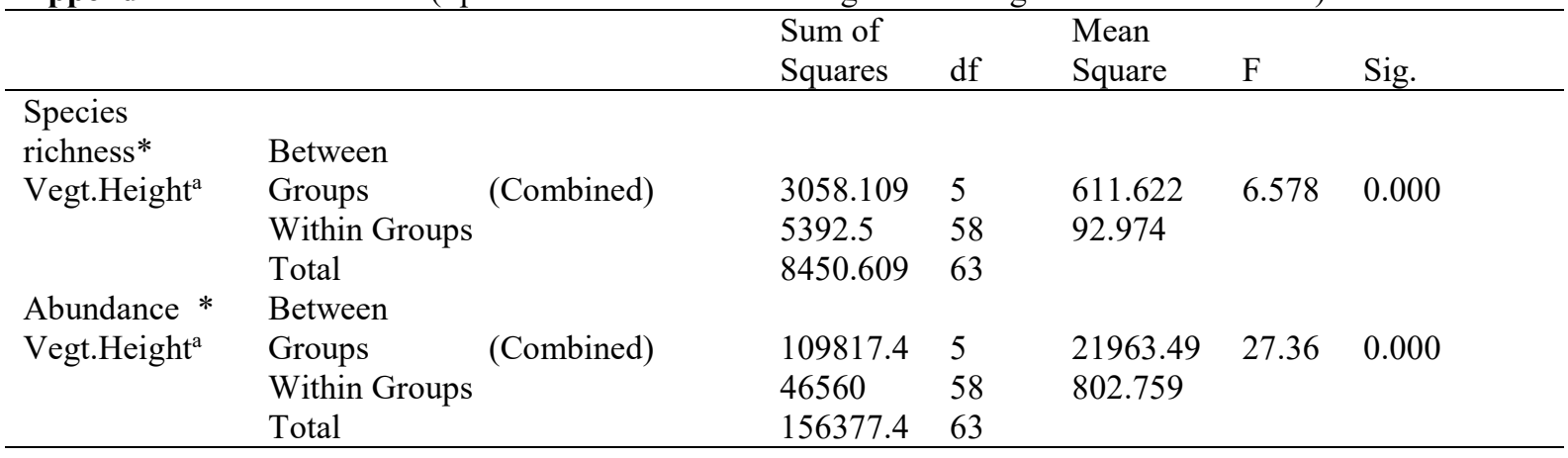


Appendix 2: ANOVA Table Species Richness and Abundance per Habitat Types of Nansebo forest

\begin{tabular}{|c|c|c|c|c|c|c|c|}
\hline & & & Sum of Squares & df & Mean Square & $\mathrm{F}$ & Sig. \\
\hline \multirow[t]{3}{*}{$\mathrm{SR} *$ Habitat } & Between Groups & (Combined) & 4788.267 & 1 & 4788.267 & 94.657 & 0.000 \\
\hline & Within Groups & & 910.533 & 18 & 50.585 & & \\
\hline & Total & & 5698.8 & 19 & & & \\
\hline \multirow[t]{3}{*}{$\mathrm{AB} *$ Habitat } & Between Groups & (Combined) & 1083.75 & 1 & 1083.75 & 0.853 & 0.368 \\
\hline & Within Groups & & 22868 & 18 & 1270.444 & & \\
\hline & Total & & 23951.75 & 19 & & & \\
\hline
\end{tabular}

